\title{
Long-range surface plasmons in dielectric-metal-dielectric structure with highly anisotropic substrates
}

\author{
Nagaraj and A. A. Krokhin \\ Department of Physics, University of North Texas, 1155 Union Circle 311427, Denton, Texas 76203, USA
}

(Received 8 October 2009; revised manuscript received 23 December 2009; published 22 February 2010)

\begin{abstract}
We present a theoretical study of long-range surface plasmons propagating in a thin metallic film clad between two identical uniaxial anisotropic dielectric crystals. We show that the proper orientation of the optical axis of the crystal with respect to the metal surface enhances the propagation length of surface plasmons. Since the proper orientation depends on surface plasmon frequency, we give the results for the propagation length in a wide range of frequencies, including the telecommunication region. To increase the role of anisotropy, we consider artificial substrates from photonic crystals, which possess an order of magnitude stronger anisotropy than the natural optical crystals. We propose Kronig-Penney model for plasmonic crystal where the substrate is a periodic sequence of dielectric delta peaks. In this model the dispersion relation for surface plasmon has a band structure where the band width tends to zero when the frequency approaches the resonant frequency.
\end{abstract}

DOI: 10.1103/PhysRevB.81.085426

PACS number(s): 42.70.Qs, 41.20.Jb, 42.25.Lc

\section{INTRODUCTION}

The efficiency of plasmonic devices is limited by finite propagation length $L(\omega)$ of surface plasmon polaritons. The main source of attenuation of propagating surface plasmon is Joule losses in the metal. The Joule losses $Q=\int \mathbf{j} \cdot \mathbf{E} d V$ are reduced if the electric field has a mode, enforced by symmetry, somewhere inside the metal plate. Since in a bulk conductor, the field decays at the skin depth $\delta$, the effect is noticeable if the plate thickness $d$ does not exceed $\delta .{ }^{1}$ Strong reduction in dissipation occurs if the plasmonic structure is symmetric, i.e., the dielectrics on both sides of the plate are the same. Then, the electric field $\mathbf{E}$ vanishes exactly at the plane of symmetry, minimizing the integral $Q .^{2}$ This plasmonic mode with antisymmetric distribution of parallel-tothe-plate component of electric field is usually called longrange surface plasmon. ${ }^{3}$ The propagation length of this mode scales as $L \sim 1 / d^{2}$ and may be as long as few millimeters or even centimeters in the near infrared ${ }^{4}$ for silver films of thickness $d \leq 50 \mathrm{~nm}$. If surface plasmon propagates along a metal strip instead of an infinite plane, some increase in propagation length can be reached for special choice of cross section of the strip. ${ }^{5}$ Short propagation length limits the size of photonic chip or component of optical circuit containing plasmonic structure. In order to reduce dissipation in plasmonic waveguides, it was proposed to groove $\mathrm{V}$-shaped channels in metal. ${ }^{6}$ These channels support propagation of long-range surface plasmons and allow experimental realization of interference, splitting, and switching of surface waves. $^{7}$

The most interesting features of surface plasmon polariton are manifested at frequencies close to the limiting frequency $\omega_{s}$ in the spectrum of surface plasmon $\omega=\omega(k)$. For the case of isotropic substrate, this frequency is given by $\omega_{s}$ $=\omega_{p} / \sqrt{1+\varepsilon}$, where $\omega_{p}$ is plasma frequency of the metal and $\varepsilon$ is the dielectric constant. Close to the resonant frequency, the surface plasmon density of states $d k / d \omega$ grows infinitely, leading to the enhancement of light emission from quantum semiconductor structures. ${ }^{8}$ Also the subwavelength resolution of plasmonic devices is strongly enhanced near the resonant frequency since the penetration depth into the dielectric vanishes at $\omega=\omega_{s} .{ }^{9}$ Unfortunately, the propagation length $L(\omega)$ quickly tends to zero near $\omega_{s}$. Because of this property, any plasmonic device cannot operate in the frequency region close to surface-plasmonic resonance. It is worthwhile to discuss here the physical reasons for such strong decay.

Propagation length, being the distance at which the energy of the wave decays by a factor of $e$, can be expressed through the rate of dissipation $Q$ and flux of energy $S(\omega)$ as follows:

$$
L(\omega)=\frac{S(\omega)}{Q(\omega)} .
$$

It is clear that the denominator $Q$, while grows smoothly with $\omega$, remains finite at any frequency. Fast decay of surface plasmon is due to vanishing of the energy flux $S$ at $\omega=\omega_{s}$. The total flux $S$ associated with propagating surface plasmon is a sum of two terms, $S=2 S_{d}+S_{m}$. Here $S_{d}$ and $S_{m}$ are Poynting vectors in one of the dielectrics and in the metal, respectively. Since the dielectric constant of the metal film is negative, $\varepsilon_{m}(\omega)<0$, the energy in the metal and in the dielectrics flows in opposite directions, i.e., $S_{d}>0$ and $S_{m}<0$. While the total flux $S$ is positive [surface plasmon is a wave with normal dispersion, $d \omega / d k>0$, if the metal (silver) film thickness is not less than $30 \mathrm{~nm}$ ], the interior of the metal gives negative contribution. It is easy to derive that $2 S_{d}+S_{m} \propto \varepsilon_{m}^{2}(\omega)-\varepsilon^{2}$. The resonant frequency is obtained from the equation $\varepsilon_{m}\left(\omega_{s}\right)+\varepsilon=0$. Therefore, the net flux $S(\omega)$ vanishes linearly at $\omega=\omega_{s}$. This simple calculation shows that the main reason for strong decay of surface plasmon near the resonant frequency is related to its dispersion but not to dissipation. For frequencies near $\omega_{s}$, the most efficient way to increase the propagation length is detuning from the resonance, which affects the numerator in Eq. (1). Thus, the most attractive for applications region of frequencies is unreachable because of very short propagation length.

At low temperatures, the Joule losses are strongly reduced due to the decrease in denominator in Eq. (1), leading to increase in the propagation length. ${ }^{10}$ At room temperatures, 
however, there are very limited options for variation in the propagation length. One of them is to affect the level of dissipation through the dielectric properties of the substrate. The field in the substrate affects the field in the metal (and thus the level of dissipation) through the boundary conditions. Surface plasmons on anisotropic lossless substrate were studied in Ref. 11. It was shown that the in-plane anisotropy gives rise to the splitting of surface plasmon by two ordinarylike and extraordinarylike surface modes, if surface plasmon propagates at an angle with respect to the optical axis. The effects of anisotropy of the substrate were studied for asymmetric plasmonic structures, i.e., for vacuum-metaldielectric structures. In Ref. 13, the proposed substrate was two-dimensional (2D) photonic crystal (a periodic arrangement of parallel cylinders) and in Ref. 12, the metallic film was deposited on multilayered dielectric heterostructure [one-dimensional (1D) photonic crystal]. In both cases an essential increase in the propagation length has been reported.

Here we consider a symmetric dielectric-metal-dielectric plasmonic structure. The advantage of this configuration is that it supports propagation of the long-range surface plasmon. The effects of anisotropy may lead to further increase in the propagation length. Indeed, we report about 20 -fold increase in the propagation length of long-range surface plasmon in the symmetric configuration when compared to that of the asymmetric (vacuum-metal-dielectric) one ${ }^{13}$ for the same given parameters at $\lambda=1.57 \mu \mathrm{m}$. It is important to mention at this point that an attempt to study the effects of anisotropy on the propagation length was made in Ref. 14 . Unfortunately, the reported results are erroneous. The calculated propagation length turned out to be as short as $10^{-15} \mathrm{~m}$, i.e., on the order of the classical electron radius. Apart from this many-orders-of-magnitude error, the reported tendency for the propagation length to grow toward the resonant frequency is wrong. It is clear from the aforementioned effect of vanishing of the total flux $S$ in Eq. (1) that even infinitesimally weak dissipation $Q$ leads to zero propagation length at $\omega=\omega_{s}$. In addition, the authors of Ref. 14 report unreasonably strong sensitivity of the propagation length to the level of anisotropy, taking into account relatively weak anisotropy they used in their calculations.

\section{CALCULATION OF THE PROPAGATION LENGTH}

The symmetric configuration that we consider consists of a metal film of thickness $d$ clad with two identical semiinfinite anisotropic dielectric substrates as shown in the inset in Fig. 1. The metal film occupies the space between $z=0$ and $z=d$ (region 1). The dielectric on top of the metal film occupies all space above $z=d$ (region 2) and the dielectric below the metal film occupies all space below $z=0$ (region 3 ). The dielectrics are assumed to be isotropic in the $x-y$ plane, i.e., $\varepsilon_{x}=\varepsilon_{y}$. The dielectric constant in the perpendicular to the interface direction (along $z$ axis) is, however, different, $\varepsilon_{z} \neq \varepsilon_{x}$.

The surface plasmon propagating along the metaldielectric interface is a $p$-polarized wave with the components of the electric field $E_{x}$ and $E_{z}$ and with the only com-

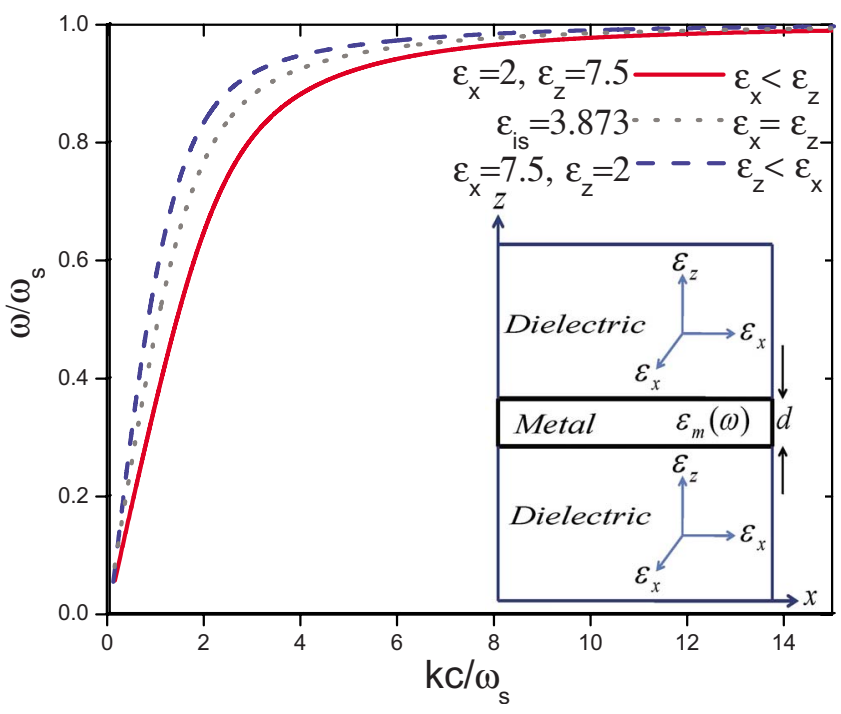

FIG. 1. (Color online) Dispersion curves for different orientations of optical axis of the anisotropic dielectric crystals with metal film of thickness $d=50 \mathrm{~nm}$. The dotted curve represents dispersion curve for equivalent isotropic dielectric with dielectric constant $\varepsilon_{i s}=\sqrt{\varepsilon_{x} \varepsilon_{z}}$.

ponent of the magnetic field $H_{y}=H(x, z)$. The field inside the metallic film is a superposition of two exponents, $H_{1}(x, z)$ $=A \exp \left(i k x+\kappa_{1} z\right)+B \exp \left(i k x-\kappa_{1} z\right)$, with $\kappa_{1}=\sqrt{k^{2}-\varepsilon_{m} \omega^{2} / c^{2}}$ being the inverse skin depth, $\kappa_{1}^{-1}=\delta$. In the substrates, which are two identical uniaxial dielectric crystals, the magnetic field is obtained from the Helmholtz equation

$$
\frac{1}{\varepsilon_{z}} \frac{\partial^{2} H}{\partial x^{2}}+\frac{1}{\varepsilon_{x}} \frac{\partial^{2} H}{\partial z^{2}}+\frac{\omega^{2}}{c^{2}} H=0
$$

The evanescent solutions of this equation are $H_{2}(x, z)$ $=C \exp \left[i k x-\kappa_{2}(z-d)\right]$ and $H_{3}(x, z)=C \exp \left(i k x+\kappa_{2} z\right)$, where $\kappa_{2}=\sqrt{\varepsilon_{x}\left(\frac{k^{2}}{\varepsilon_{z}}-\frac{\omega^{2}}{c^{2}}\right)}$ is the inverse decay length of the surface plasmon field in dielectric, and $\varepsilon_{m}(\omega)=1-\omega_{p}^{2} / \omega^{2}$. From the continuity of the magnetic field $H(x, z)$ and electric field $E_{x}(x, z)=\left(c / i \omega \varepsilon_{x}\right) \partial H / \partial z$ at the interfaces $z=0$ and $z=d$, we obtain the following dispersion equation for the surface plasmon:

$$
\frac{\kappa_{2} \varepsilon_{m}}{\kappa_{1} \varepsilon_{x}}=-\tanh \left(\frac{\kappa_{1} d}{2}\right) .
$$

This equation gives the dispersion for the mode with antisymmetric distribution of $E_{x}$ with respect to the plane of symmetry $z=d / 2$, i.e., for the so-called long-range surface plasmon. It is easy to see that interchanging the values of $\varepsilon_{x}$ and $\varepsilon_{z}$, two different dispersion equations are obtained. The transformation $\varepsilon_{x} \leftrightarrow \varepsilon_{z}$ means $90^{\circ}$ rotation of the optical axis of the dielectric crystal with respect to the metal surface. In Fig. 1 the solid and dashed curves are the dispersion Eq. (3) for two different orientations. Both the curves approach the same resonant frequency 


$$
\omega_{s}=\frac{\omega_{p}}{\sqrt{1+\sqrt{\varepsilon_{x} \varepsilon_{z}}}}
$$

at $k \rightarrow \infty$. Since $\omega_{s}$ is symmetric with respect to $\varepsilon_{x}$ and $\varepsilon_{z}$, the equivalent isotropic dielectric constant may be defined as $\varepsilon_{i s}=\sqrt{\varepsilon_{x} \varepsilon_{z}}$. The dispersion curve for the structure with equivalent isotropic dielectrics (dotted line) lies in between the solid and the dashed lines in Fig. 1. The dispersion curves are plotted for silver film of thickness of $d=50 \mathrm{~nm}$. The numerical values of the principal dielectric constants are taken to be 2 and 7.5. These are the homogenized values obtained in the low-frequency limit for photonic crystal of $\mathrm{Si}$ cylinders arranged in a square lattice with Si filling of $57 \% .^{15}$ The larger value 7.5 corresponds to the dielectric constant along the cylinders. In the plane of periodicity, the photonic crystal is isotropic with dielectric constant equals 2 .

The asymmetry of the dispersion Eq. (3) with respect to the interchange $\varepsilon_{x} \leftrightarrow \varepsilon_{z}$ gives rise to different propagation lengths for two different orientations. Since only the case of weak dissipation is of practical interest, we consider the region of frequencies where the imaginary part of the dielectric function of the metal film $\varepsilon_{m}(\omega)=\varepsilon_{m}^{\prime}(\omega)+i \varepsilon_{m}^{\prime \prime}(\omega)$ is small, i.e., $\varepsilon_{m}^{\prime \prime} \ll \varepsilon_{m}^{\prime}$. For a typical metal this condition is valid in the infrared and visible regions. In weakly dissipative medium, the wave vector acquires small imaginary part, $k=k^{\prime}+i k^{\prime \prime}$. Expanding the dispersion Eq. (3) over $\varepsilon_{m}^{\prime \prime}$ and $k^{\prime \prime}$ and keeping the linear terms, the following result for the propagation length $L(\omega)=1 / 2 k^{\prime \prime}$ is obtained:

$$
\begin{aligned}
L(\omega)= & \frac{k}{2 \varepsilon_{m}^{\prime \prime}} \frac{1}{\kappa_{2}^{2}}\left[\frac{\varepsilon_{x}}{\varepsilon_{z}}-\frac{\kappa_{2}^{2}}{\kappa_{1}^{2}}+d \frac{\kappa_{2}}{2}\left(\frac{\left|\varepsilon_{m}\right|}{\varepsilon_{x}} \frac{\kappa_{2}^{2}}{\kappa_{1}^{2}}-\frac{\varepsilon_{x}}{\left|\varepsilon_{m}\right|}\right)\right] \\
& \times\left[\frac{1}{\left|\varepsilon_{m}\right|}-\frac{\omega^{2}}{2 c^{2} \kappa_{1}^{2}}+\frac{\omega^{2}}{4 c^{2}} \frac{d}{\kappa_{2}}\left(\frac{\left|\varepsilon_{m}\right|}{\varepsilon_{x}} \frac{\kappa_{2}^{2}}{\kappa_{1}^{2}}-\frac{\varepsilon_{x}}{\left|\varepsilon_{m}\right|}\right)\right] .
\end{aligned}
$$

Apart from the factor $1 / \varepsilon_{m}^{\prime \prime}$, all the quantities on the righthand side (rhs) are calculated for a lossless metal, $\varepsilon_{m}^{\prime \prime}=0$. The propagation length $L(\omega) \rightarrow 0$ when $\omega \rightarrow \omega_{s}$. As it was mentioned in the introduction, this effect is due to surface plasmon dispersion (vanishing of the group velocity) but not due to increase in dissipation. The dispersion equation Eq. (3) and the propagation length Eq. (5) can be simplified in the limiting case of thick film.

The limit $d \rightarrow \infty$ is readily obtained from Eqs. (3) and (5). In this limit the surface plasmons propagating along two metal-dielectric interfaces do not interact with each other, therefore the dispersion relation is reduced to the result obtained in Ref. 3 for semi-infinite metal

$$
k=\frac{\omega}{c} \sqrt{\varepsilon_{z}\left|\varepsilon_{m}\right|}\left(\frac{\left|\varepsilon_{m}\right|+\varepsilon_{x}}{\varepsilon_{m}^{2}-\varepsilon_{x} \varepsilon_{z}}\right)^{1 / 2} .
$$

Now, using Eq. (6), we obtain from Eq. (5) the following result for the propagation length:

$$
L(\omega)=\frac{c}{\omega \varepsilon_{m}^{\prime \prime}} \frac{\sqrt{\varepsilon_{z}\left|\varepsilon_{m}\right|}}{\varepsilon_{x} \varepsilon_{z}} \frac{\left(\varepsilon_{m}^{2}-\varepsilon_{x} \varepsilon_{z}\right)^{3 / 2}\left(\left|\varepsilon_{m}\right|+\varepsilon_{z}\right)^{1 / 2}}{\left(\varepsilon_{m}^{2}+\varepsilon_{x} \varepsilon_{z}+2 \varepsilon_{z}\left|\varepsilon_{m}\right|\right)} .
$$

Of course, in the case of isotropic dielectrics, $\varepsilon_{x}=\varepsilon_{z}$, Eqs. (3)-(8) are reduced to the well-known results. ${ }^{3,16-21}$

The calculation of propagation length in the limit when $d \rightarrow \infty$ and in the limit when $\omega \rightarrow \omega_{s}$ can both be considered equivalent because at frequencies close to resonant frequency $\omega_{s}$, the decay length $\kappa_{2}^{-1} \rightarrow 0$ (since the surface plasmon wave vector $k \rightarrow \infty)$. For the evanescent field of surface plasmon, this has the same effect as having a semi-infinite metal film. Consequently, the propagation length $L(\omega) \sim\left(\omega_{s}-\omega\right)^{3 / 2}$ for a metal film of any given thickness close to resonant frequency, as one obtains from Eq. (7).

In the opposite limit of thin film, $d \rightarrow 0$, Eq. (5) is simplified in the long-wavelength regime, i.e., when surface plasmon behaves like a transverse light wave

$$
L(\omega)=\frac{2}{\varepsilon_{m}^{\prime \prime} d^{2}}\left(\frac{c}{\omega}\right)^{3} \frac{\left|\varepsilon_{m}\right|^{3}}{\varepsilon_{x} \varepsilon_{z}^{3 / 2}\left(\left|\varepsilon_{m}\right|+\varepsilon_{z}\right)} .
$$

In this approximation the dispersion relation $\omega \approx k c / \sqrt{\varepsilon_{z}}$ does not contain characteristics of the metal but the propagation length does. It follows from Eq. (8) that for fixed $\omega$, the propagation length scales as $d^{-2}$, which is a signature of the long-range surface plasmon. Analysis of the long-wavelength limit shows that the favorable orientation in the limit of thin film $(d \rightarrow 0)$ is not the same as the one in the case of a semi-infinite film $(d \rightarrow \infty)$. In the limit of thin film (for a given frequency), $[L(\omega)]^{-1} \propto \varepsilon_{x} \varepsilon_{z}^{3 / 2}$, while in the case of a semi-infinite film, $[L(\omega)]^{-1} \propto \varepsilon_{x} \varepsilon_{z}^{1 / 2}$. There is a certain critical frequency $\omega_{c r}$ (for a given thickness $d$ of metal film) at which the favorable orientation reverses (see Fig. 2). The dependence of critical frequency on the thickness $d$ of the metal film is discussed in more detail later in this paper.

Further examination of asymptotes in the linear dispersion regime $\left(\omega \approx k c / \sqrt{\varepsilon_{z}}\right)$ reveals some interesting details. The asymptote in the limit of thin film $(k d \ll 1)$ is given by Eq. (8), and in the limit of thick film $(k d \gg 1)$, we obtain from Eq. (5)

$$
L(\omega) \approx \frac{\left|\varepsilon_{m}\right|}{\varepsilon_{x} \varepsilon_{m}^{\prime \prime} k} \frac{\left(\varepsilon_{m}^{2}-\varepsilon_{x} \varepsilon_{z}\right)}{\left(\left|\varepsilon_{m}\right|+2 \varepsilon_{z}\right)}, \quad k d \gg 1 .
$$

The linear dispersion regime in the limit of very thin metal film spans over a broad range of frequencies, while in the case of very thick metal film, the linear dispersion regime is limited only to low frequencies (see Fig. 3). Although very thin film favors a much longer propagation length (see Figs. 2 and 4), it is relatively more difficult to reach the resonance frequency $\omega_{s}$ (see Fig. 3). In addition to this, the surface plasmon density of states $d k / d \omega$ for very thin films is relatively very low (due to the greater slope $d \omega / d k$ of the dispersion curve), leading to the decrease in probability of light emission. We note that in the limit of very thick metal, the form of Eq. (9) is different from Eq. (7), because, linear dispersion does not span over a broad frequency range as compared to linear dispersion in the limit of very thin metal. However, at very low frequencies (in the long wavelength regime), both Eqs. (7) and (9) are reduced to 

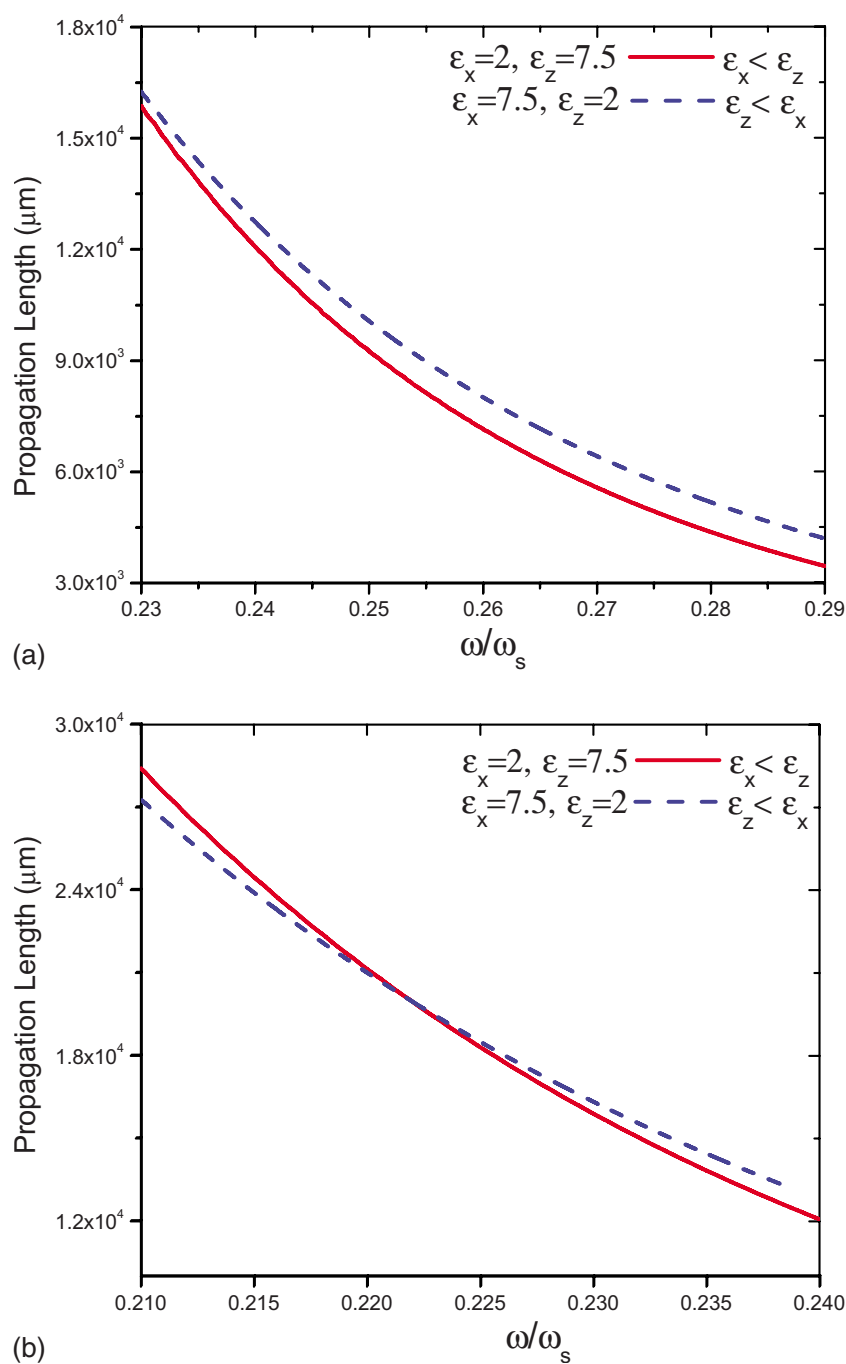

FIG. 2. (Color online) The top figure shows propagation length for parallel $\left(\varepsilon_{x}>\varepsilon_{z}\right)$ and perpendicular $\left(\varepsilon_{x}<\varepsilon_{z}\right)$ orientations for metal thickness $d=20 \mathrm{~nm}$. The bottom figure shows the same curves between $\omega=0.21 \omega_{s}$ and $\omega=0.24 \omega_{s}$.

$$
L(\omega) \approx \frac{\varepsilon_{m}^{2}}{\varepsilon_{x} \varepsilon_{m}^{\prime \prime} k}, \quad k d \gg 1 .
$$

The propagation length curves [obtained from Eq. (5)] for the two orientations $\left(\varepsilon_{x}<\varepsilon_{z}\right.$ and $\left.\varepsilon_{z}<\varepsilon_{x}\right)$ and for the equivalent isotropic case $\left(\varepsilon_{x}=\varepsilon_{z}=\varepsilon_{i s}\right)$ are shown in Fig. 4. It is clear from Eq. (5) and Fig. 4 that the effect of anisotropy of the dielectric may be used as an advantage to modulate the propagation length by changing the orientation of the optical axis of the dielectric crystal. The decreasing trend of $L(\omega)$ toward higher frequencies can be seen in Fig. 4, where $\omega$ $=0.36 \omega_{s}$ corresponds to $\lambda=0.961 \mu \mathrm{m}$, and $\omega=0.45 \omega_{s}$ corresponds to $\lambda=0.768 \mu \mathrm{m}$. This range is much below the telecommunications wavelength $(1.3-1.6 \mu \mathrm{m})$. Though the isotropic case is more favorable for wavelengths between $\lambda=0.768 \mu \mathrm{m}$ and $\lambda=0.961 \mu \mathrm{m}$, it is obvious from Fig. 4 that the perpendicular orientation $\left(\varepsilon_{x}<\varepsilon_{z}\right)$ is most preferable at wavelengths above $\lambda=0.961 \mu \mathrm{m}$. However, at frequencies higher than $\omega_{c r}$ (critical frequency at which the favor-
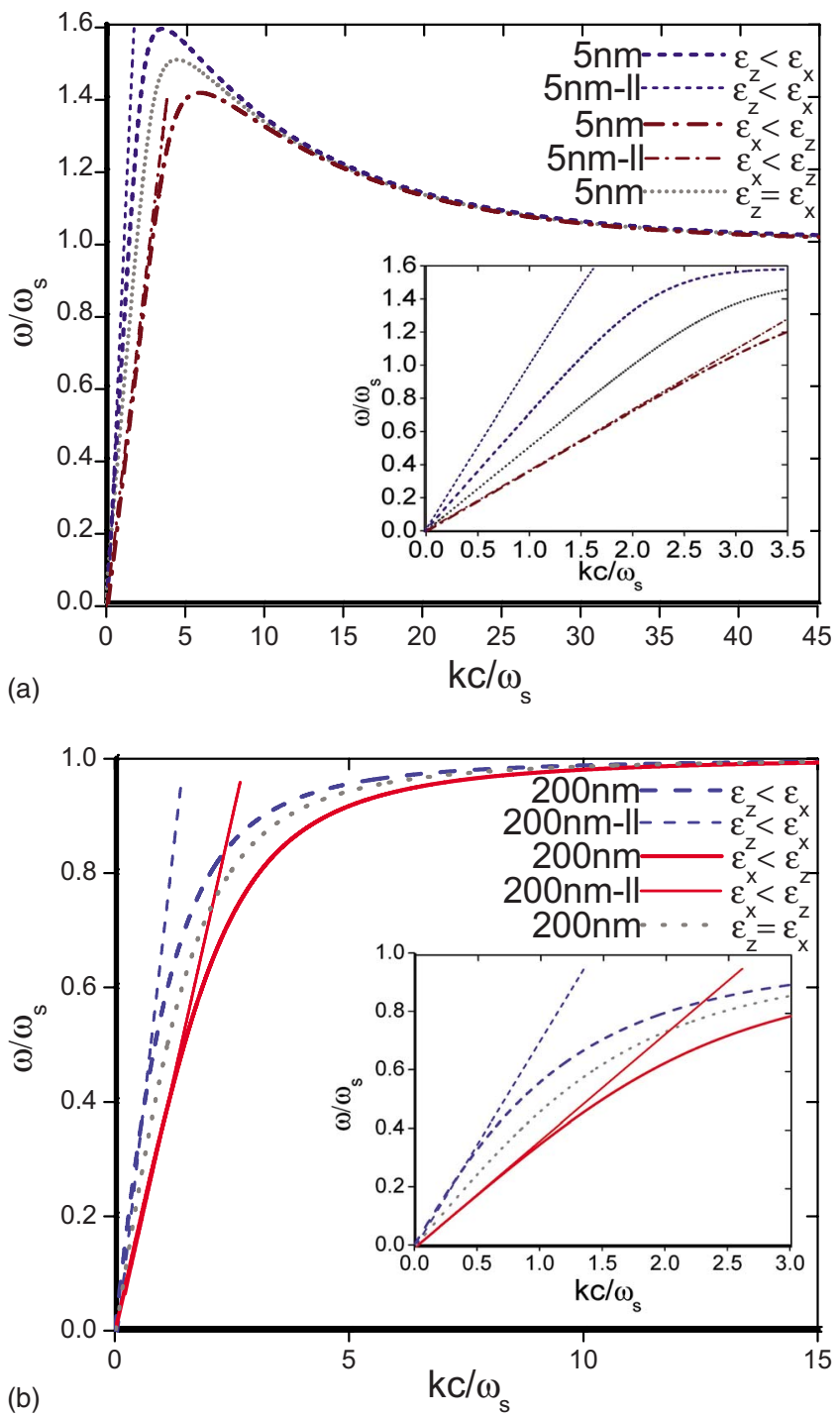

FIG. 3. (Color online) Dispersion curves and light lines (11) for different orientations for metal thicknesses $d=5 \mathrm{~nm}$ and $d=200 \mathrm{~nm}$. Light lines for equivalent isotropic case $\left(\varepsilon_{x}=\varepsilon_{z}\right)$ are not included in the figure. The anomalous dispersion curves correspond to metal thickness $d=5 \mathrm{~nm}$ (top figure).

able orientation reverses), the parallel orientation is more preferable.

For silver metal film of thickness $d=50 \mathrm{~nm}$, and for the dielectrics having principal dielectric constants as 2 and 7.5, this critical frequency $\omega_{c r}$ occurs at $\omega \approx 0.4 \omega_{s}$, which corresponds to $\lambda_{c r}=0.865 \mu \mathrm{m}$, i.e., it is still much below the telecommunications wavelength.

In Fig. 5, the critical frequency is plotted as a function of thickness $d$ of the metal film. The ratio $\omega_{c r} / \omega_{s}$ gradually increases with $d$ until it saturates at $\omega \approx 0.62 \omega_{s}$, which corresponds to thickness $d=200 \mathrm{~nm}$. The saturation of curve in Fig. 5 indicates that for a given anisotropy of dielectric, the metal thickness of $d=200 \mathrm{~nm}$ can be safely considered as semi-infinite. This fact is clearly demonstrated in the inset of Fig. 5, where the difference between propagation lengths [calculated from the "exact" Eq. (5) and it's asymptote $\left[L_{\infty}(\omega), d=\infty\right]$ Eq. (7) $]$ as a function of frequency is plotted. 


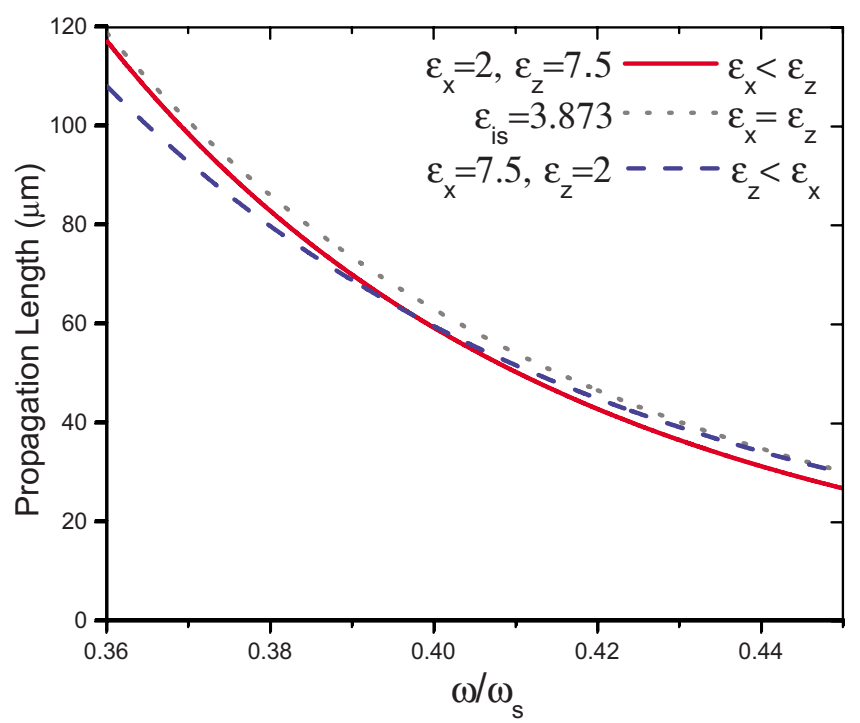

FIG. 4. (Color online) Propagation length for parallel, perpendicular orientations, and for the equivalent isotropic case with metal film of thickness $d=50 \mathrm{~nm}$. $\omega=0.36 \omega_{s}$ corresponds to $\lambda=0.961 \mu \mathrm{m}$ and $\omega=0.45 \omega_{s}$ corresponds to $\lambda=0.768 \mu \mathrm{m}$.

It is to be noted that for the given orientation $\left(\varepsilon_{x}<\varepsilon_{z}\right)$, the propagation length at $\omega=0.2 \omega_{s}$ is about $700 \mu \mathrm{m}$, as compared to the difference $L(\omega)-L_{\infty}(\omega)$ of $18 \mu \mathrm{m}$ only. The plot in the inset shows the strong agreement between Eqs. (5) and (7). It is also obvious from Fig. 5 that the critical frequency $\omega_{c r}$ shifts toward lower frequencies as the metal thickness $d$ decreases. This behavior strongly influences the favorability of one orientation over the other. In particular, within the telecommunication bandwidth (between $\omega \approx 0.22 \omega_{s}$ ) and $\omega \approx 0.25 \omega_{s}$, having $\varepsilon_{z}<\varepsilon_{x}$ is more preferable for very thin metal, whereas having $\varepsilon_{x}<\varepsilon_{z}$ is more preferable for a thick metal (compare Figs. 2 and 6).

In Fig. 6, the propagation length for $\varepsilon_{x}<\varepsilon_{z}, \varepsilon_{x}=\varepsilon_{z}$, and $\varepsilon_{z}<\varepsilon_{x}$ is plotted. The frequency range from $\omega=0.22 \omega_{s}$

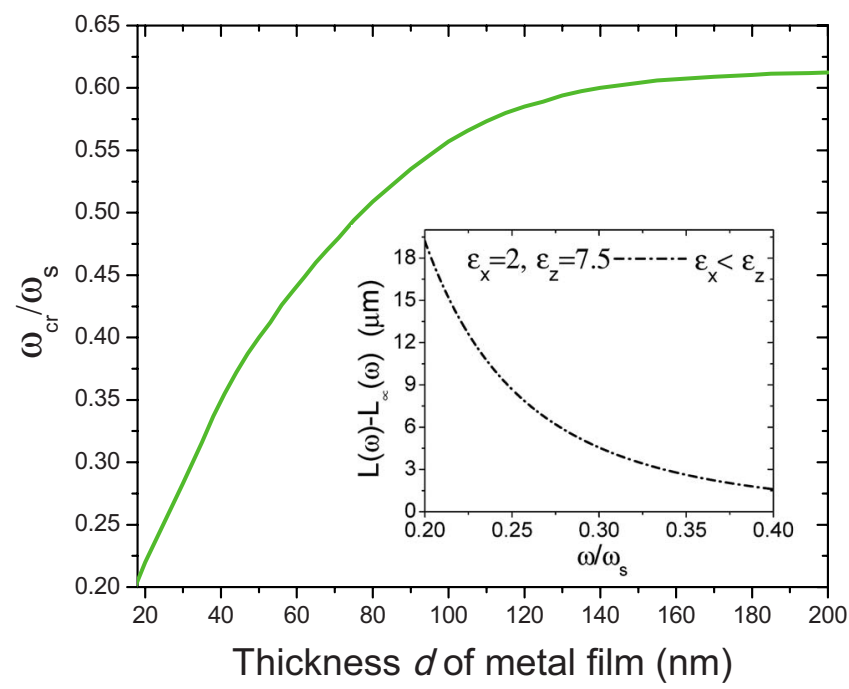

FIG. 5. (Color online) $\omega_{c r} / \omega_{s}$ as a function of thickness $d$ of the metal film. The inset shows the difference between propagation lengths (obtained using Eqs. (5) and (7)) as a function of frequency for metal thickness $d=200 \mathrm{~nm}$.

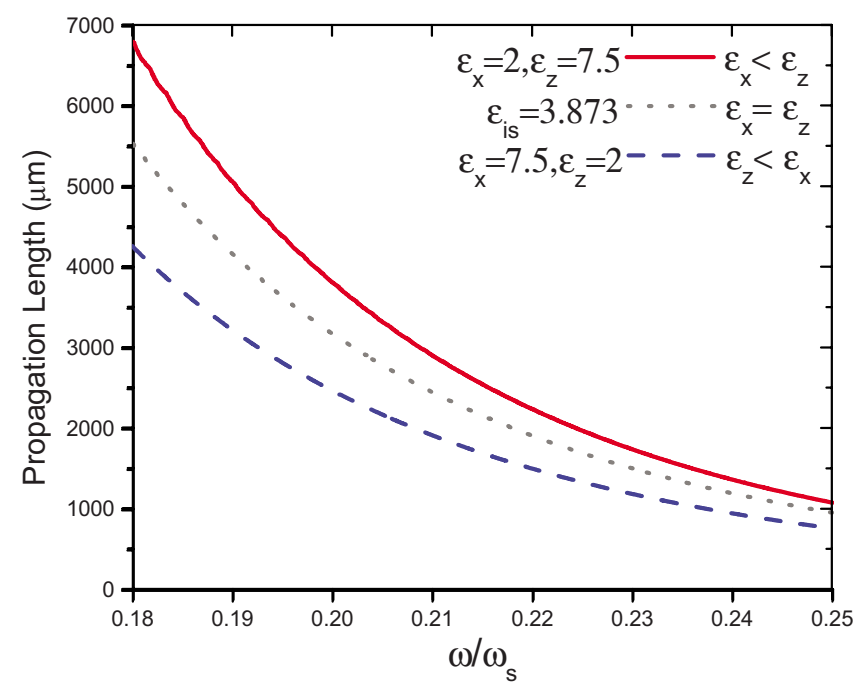

FIG. 6. (Color online) Propagation length $L(\omega)$ for different orientations of dielectric crystal with metal film of thickness $d=50 \mathrm{~nm}$.

$(\lambda=1.57 \mu \mathrm{m})$ to $\omega=0.25 \omega_{s}(\lambda=1.384 \mu \mathrm{m})$ contains the telecommunications bandwidth. Clearly, the propagation length is enhanced by more than $50 \%$ when the optical axis of uniaxial dielectric substrate is aligned in the direction perpendicular to the interface. In particular, if the substrates are 2D photonic crystals, the preferable orientation of cylinders is perpendicular to the metal film.

Interestingly, the propagation length of surface plasmons for symmetric configuration is one order of magnitude higher than the propagation length of surface plasmons for asymmetric configuration. ${ }^{13}$ For instance, $L(\omega) \approx 2.3 \mathrm{~mm}$ at $\lambda=1.57 \mu \mathrm{m}$ for perpendicular orientation in the case of symmetric configuration compared to $L(\omega) \approx 100 \mu \mathrm{m}$ at $\lambda=1.57 \mu \mathrm{m}$ for the same orientation for asymmetric configuration. That is an enhancement of propagation length by more than 20 times. It means that the propagation length (in the case of symmetric configuration that we considered in this paper) is on the order of a few millimeters within the telecommunications bandwidth (see Fig. 6).

Another important characteristic of plasmonic structure is penetration depth $\left(\kappa_{2}^{-1}\right)$. As the penetration depth determines the coupling strength of the surface plasmons with other elements of integrated photonic circuitry, an enhancement in penetration depth is desirable. Indeed in Fig. 7, the penetration depth for perpendicular orientation is enhanced by more than $300 \%$ compared to the penetration depth for parallel orientation, reasserting the requirement that the perpendicular orientation is more preferable for bandwidths corresponding to the telecommunications wavelength.

\section{KRONIG-PENNEY MODEL FOR PLASMONIC CRYSTAL}

Homogenization of photonic crystals, which we propose to use as anisotropic substrate, has been developed for the bulk modes. Currently there are several approaches, apart from the one proposed in Ref. 15 to the problem of homog- 


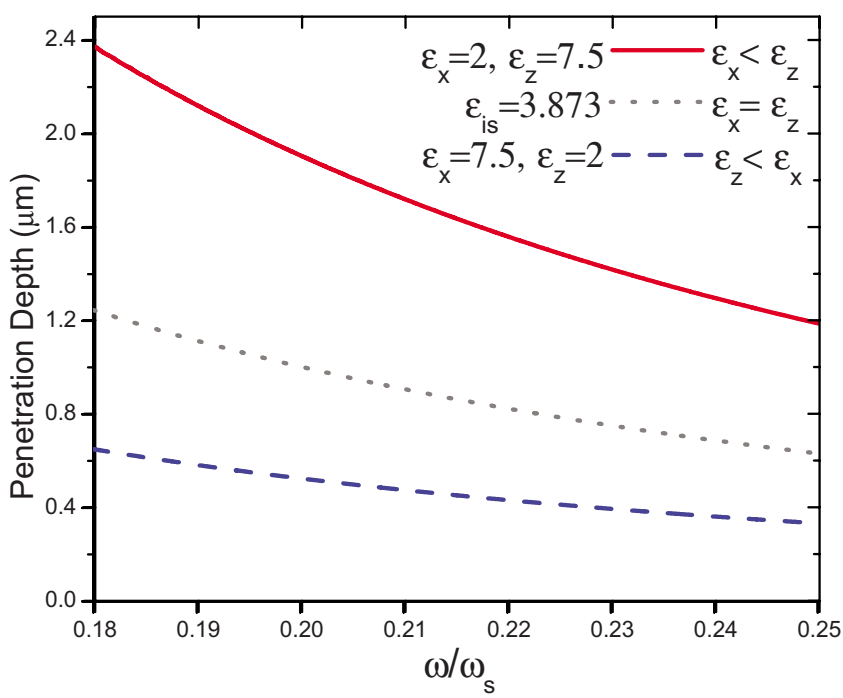

FIG. 7. (Color online) Penetration depth of surface plasmons for different orientations of dielectric crystal with metal film of thickness $d=50 \mathrm{~nm}$.

enization of metamaterials. Being technically different, however, they lead to the equivalent results for the effective dielectric constants in the low-frequency region away from internal resonances, see, e.g. ${ }^{22}$ It is not clear, however, whether the "bulk" effective dielectric constants can be used in the case of surface modes which are strongly inhomogeneous in the direction perpendicular to the interface. This inhomogeneity may also enhance the contribution of higher harmonics to the dissipation and, thus, reduce the propagation length in Eq. (5). The latter was calculated assuming that the substrate is a homogeneous anisotropic dielectric, i.e., all the spacial harmonics of the electromagnetic field of surface plasmon have been ignored. To clarify the possibility of homogenization of plasmonic crystal and evaluate the contribution of higher harmonics, we consider here a simple KronigPenney model. Usually, a periodic structure in a plasmonic crystal is associated with a periodic dielectric pattern on the surface of a metal film. ${ }^{23}$ Here we consider a periodic arrangement of infinitesimally thin dielectric sheets parallel to axis $y$ and oriented perpendicular to semi-infinite metal, see Fig. 8. The dielectric constant of this structure can be represented as a series of delta peaks

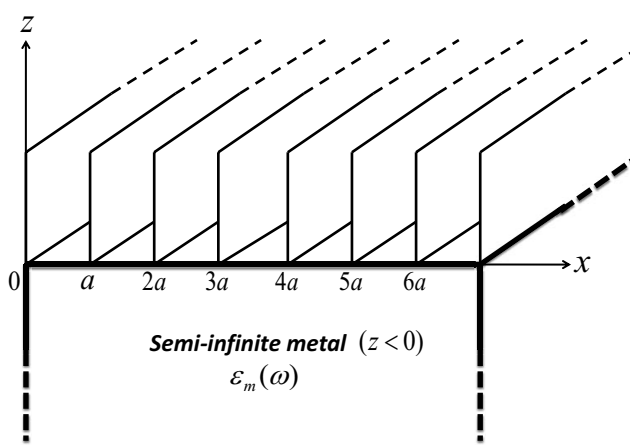

FIG. 8. Kronig-Penney model for plasmonic crystal.

$$
\varepsilon(x)=1+\varepsilon_{0} a \sum_{n} \delta(x-n a), \quad z>0 .
$$

In the long-wavelength limit this substrate behaves as a uniaxial optical crystal with effective dielectric constants

$$
\begin{gathered}
\varepsilon_{z}=\varepsilon_{y}=\frac{1}{a} \int_{-a / 2}^{a / 2} \varepsilon(x) d x=1+\varepsilon_{0}, \\
\frac{1}{\varepsilon_{x}}=\frac{1}{a} \int_{-a / 2}^{a / 2} \frac{1}{\varepsilon(x)} d x=1 .
\end{gathered}
$$

The magnetic component $H(x, z)$ of surface plasmon field decays exponentially away from the interface: in the metal $(z<0), \quad H(x, z)=\exp \left(\kappa_{1} z-i \omega t\right) h(x)$ and in the substrate $(z>0), \quad H(x, z)=\exp \left(-\kappa_{2} z-i \omega t\right) h(x)$. The continuity of $H(x, z)$ at $z=0$ is satisfied and within the interval $-a / 2<x<a / 2$ the function $h(x)$ is a superposition of two plane waves

$$
h(x)=A e^{i q x}+B e^{-i q x},
$$

where

$$
q^{2}=\kappa_{1}^{2}-(\omega / c)^{2}\left|\varepsilon_{m}(\omega)\right|=\kappa_{2}^{2}+(\omega / c)^{2} .
$$

The values of $h(x)$ on the whole axis $x$ are obtained from the Bloch theorem, $h(x+a)=\exp (i k x) h(x) . h(x)$ is a continuous function at $x=0$ but its derivative has a discontinuous jump

$$
h^{\prime}(+0)-h^{\prime}(-0)=-\varepsilon_{0} a \frac{\omega^{2}}{c^{2}} h(0) .
$$

The continuity of $h(x)$ at $x=0$ together with Eq. (16) form a set of homogeneous equations for $A$ and $B$. Equating its determinant to zero, the following dispersion equation is obtained:

$$
\cos k a=\cos q a-\frac{\varepsilon_{0} a^{2}}{2} \frac{\omega^{2}}{c^{2}} \frac{\sin q a}{q a} .
$$

In this equation the wave vector $q$ is a function of frequency $\omega$. The dependence $q(\omega)$ is obtained from the continuity of the tangential electric field $E_{x}=-(i c / \omega \varepsilon) \partial H / \partial z$ at $z=0$. This condition gives

$$
\kappa_{1}=\left|\varepsilon_{m}(\omega)\right| \kappa_{2} .
$$

Equations (15) and (18) determine the frequency dependencies $\kappa_{1}(\omega), \kappa_{2}(\omega)$, and $q(\omega)$

$$
\begin{aligned}
& \kappa_{1}^{2}=\frac{\omega^{2}}{c^{2}} \frac{\varepsilon_{m}^{2}(\omega)}{\left|\varepsilon_{m}(\omega)\right|-1}, \\
& \kappa_{2}^{2}=\frac{\omega^{2}}{c^{2}} \frac{1}{\left|\varepsilon_{m}(\omega)\right|-1}, \\
& q^{2}=\frac{\omega^{2}}{c^{2}} \frac{\left|\varepsilon_{m}(\omega)\right|}{\left|\varepsilon_{m}(\omega)\right|-1} .
\end{aligned}
$$

Since $\left|\varepsilon_{m}(\omega)\right|>1$, the following inequality holds, $\kappa_{2}<q<\kappa_{1}$. When $\omega \rightarrow \omega_{p} / \sqrt{2}$ all three parameters $\kappa_{1}, \kappa_{2}$, 


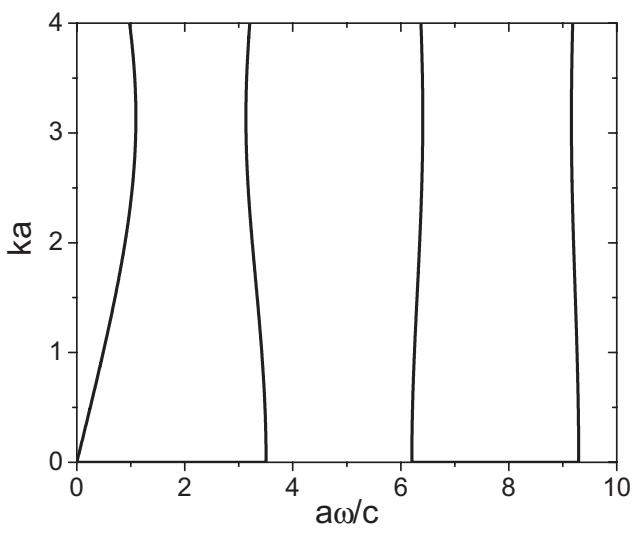

FIG. 9. The first four allowed bands for a plasmonic crystal of Si semispace and periodic substrate with $\varepsilon_{0}=3$.

and $q$ diverge. As one can see from Eq. (17) the Bloch vector $k$ also formally diverges. The frequency $\omega_{s}=\omega_{p} / \sqrt{2}$ is the limiting frequency in the dispersion Eq. (17). It is the resonant frequency of the plasmonic crystal. The resonant frequency is independent on the details of the substrate since in the Kronig-Penney model the filling fraction of the dielectric component is exactly zero. Near the resonance the wavelength $2 \pi / k$ vanishes, therefore the field of the surface plasmon is insensitive to the presence of the dielectric sheets, which are separated by a finite distance $a$, i.e., by infinite number of wavelength. In the case of nonzero filling fraction of the dielectric component the resonant frequency depends on the properties of the substrate. ${ }^{24}$

The structure of the low-frequency bands of Eq. (17) is similar to that of any periodic system. Few first bands are shown in Fig. 9. Unlike standard Kronig-Penney model, the width of the allowed bands decreases with frequency. Near the resonant frequency the spectrum becomes pointlike. Zero-width frequency bands are accumulated toward $\omega_{p} / \sqrt{2}$. Here the spectrum becomes dense and asymptotically it coincides with the spectrum of surface plasmon near its resonant frequency. This tendency is clearly seen in Fig. 10.

Long wavelength limit. In the limit $k a, q a \ll 1 \mathrm{Eq}$. (17) is simplified

$$
k^{2}=\frac{\omega^{2}}{c^{2}}\left[\frac{\left|\varepsilon_{m}(\omega)\right|}{\left|\varepsilon_{m}(\omega)\right|-1}+\varepsilon_{0}\right] .
$$

Dispersion of surface plasmon with anisotropic homogeneous substrate is given by Eq. (6). Substituting in Eq. (6) the effective dielectric constants of the substrate given by Eqs. (12) and (13) the following dispersion equation is obtained:

$$
k^{2}=\frac{\omega^{2}}{c^{2}} \frac{\left(1+\varepsilon_{0}\right)\left|\varepsilon_{m}(\omega)\right|\left[1+\left|\varepsilon_{m}(\omega)\right|\right]}{\varepsilon_{m}^{2}-\left(1+\varepsilon_{0}\right)} .
$$

In the limit $\omega \rightarrow 0$ Eqs. (22) and (23) have the same linear asymptotic, $k=(\omega / c) \sqrt{1+\varepsilon_{0}}$. This means that in the lowfrequency limit the Kronig-Penney plasmonic crystal homogenizes and the effective dielectric constants coincide with those obtained for 1D photonic crystal. Two dispersion curves corresponding to Eqs. (22) and (23) are shown in Fig.

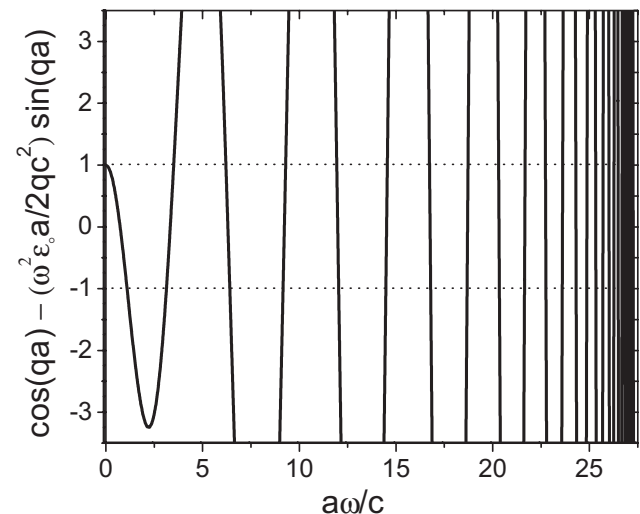

FIG. 10. The rhs of Eq. (17) vs frequency. The band edges are the intersections of the curve with two horizontal lines at the level \pm 1 .

11. In fact, the dispersion curves in Fig. 11 are very close to each other not only in the low-frequency limit but within a wide range of frequencies. This means that the substrate can be considered as an effective homogeneous medium once the conditions $q a, k a \ll 1$ are satisfied. Two dispersion curves exhibit essential difference only near the resonant frequencies $\omega_{p} / \sqrt{2}$ and $\omega_{s}=\omega_{p} / \sqrt{1+\varepsilon_{0}}=\omega_{p} / 2$. Here $q, k \rightarrow \infty$ and the dispersion Eq. (22) is not valid since the conditions $q a, k a \ll 1$ cannot be satisfied.

Contribution of higher harmonics. The contribution of higher harmonics is evaluated from the Fourier expansion of magnetic field

$$
h(x)=\exp (i k x) f_{k}(x)=\exp (i k x) \sum_{n} f_{k}(n) \exp (2 \pi i n x / a) .
$$

The distribution of the field $h(x)$ within a period $-a / 2<x<a / 2$ is obtained from Eq. (14), where the ratio

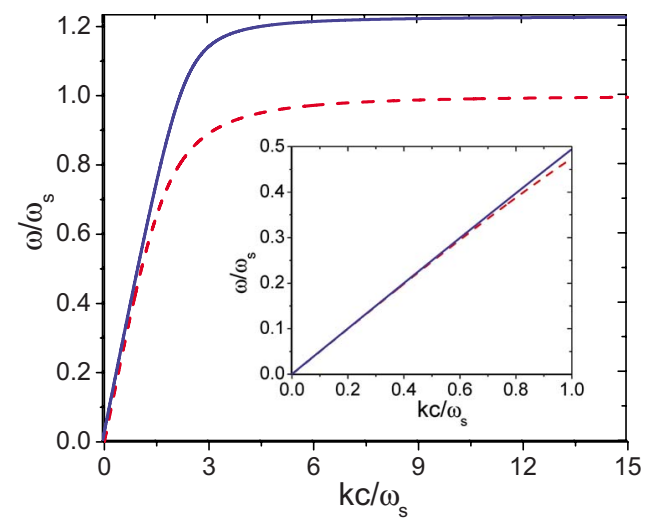

FIG. 11. (Color online) Dispersion curves for plasmonic crystal with $\varepsilon_{0}=3$ in the limit $k a, q a \ll 1$ [Eq. (22), solid line] and for plasmonic structure with a homogeneous dielectric substrate with $\varepsilon_{x}=1$ and $\varepsilon_{z}=1+\varepsilon_{0}$ [Eq. (23), dashed line]. The inset shows the region of low frequencies. The frequency is normalized to $\omega_{s}=\omega_{p} / \sqrt{1+\varepsilon_{0}}=\omega_{p} / 2$. 


$$
\frac{B}{A}=-\frac{e^{i q a}-e^{i k a}}{e^{-i q a}-e^{i k a}}
$$

Normalizing the magnetic field by the condition $h(0)=1$, we obtain the following result:

$$
h(x)=\left[e^{i k a} \sin q x+\sin q(a-x)\right] / \sin q a, \quad-a / 2<x<a / 2 .
$$

The Fourier harmonics are easily calculated from Eqs. (24) and (26)

$$
\begin{aligned}
f_{k}(n)= & \frac{(-1)^{n}}{i a \sin q a}\left[\left(e^{i k a}-e^{-i q a}\right) \frac{\sin [a(q-k) / 2]}{q-k-2 \pi n / a}+\left(e^{i q a}\right.\right. \\
& \left.\left.-e^{i k a}\right) \frac{\sin [a(q+k) / 2]}{q+k+2 \pi n / a}\right] .
\end{aligned}
$$

In the long wavelength limit $k a \ll 1$ all the harmonics vanish but the one with $n=0$

$$
f_{k}(n) \approx(-1)^{n+1}\left[\frac{a(q-k)}{2 \pi n}\right]^{2}, \quad n \neq 0 .
$$

Thus, the contribution of higher harmonics to the electromagnetic field of surface plasmon decays as $a^{2}(k-q)^{2}$. This justifies the approximation of the effective medium theory in calculation of the propagation length of surface plasmon Eq. (7) propagating along a photonic crystal substrate.

\section{CONCLUSIONS}

We have studied the propagation of long-range surface plasmons in a dielectric-metal-dielectric structure with highly anisotropic substrates. We have derived the formula for propagation length valid in a wide range of frequencies, including the telecommunication region, and have shown that the proper orientation of optical axis of uniaxial dielec- tric substrates with respect to the metal surface enhances the propagation length as well as the penetration depth of surface plasmons. The frequency $\omega_{c r}$ [at which $L(\omega)$ is independent of the orientation of optical axis] shifts toward lower frequencies for thinner metal films indicating that the parallel orientation $\left(\varepsilon_{z}<\varepsilon_{x}\right)$ becomes increasingly favorable as thickness $d$ of the metal film is reduced. We demonstrated an important general property of long-range surface plasmons that the propagation length tends to zero close to resonance frequency $\omega_{s}$ not because of increase in dissipation but due to the compensation of electromagnetic energy fluxes in the metal and in the dielectrics. This is a direct consequence of dispersion resulting from the negative value of the dielectric constant of metal. We have also shown that the propagation length for symmetric configuration is one order of magnitude greater than the propagation length for asymmetric configuration. This enhancement in propagation length can cut the limitation on the size of photonic chip or component of optical circuit containing plasmonic structure. We proposed a simple, analytically solvable Kronig-Penney model for plasmonic crystal. The obtained dispersion equation has a band structure. In the long-wavelength limit this equation is reduced to the equation obtained for surface plasmon propagating along a homogeneous anisotropic substrate. This result justifies application of the homogenization procedure for surface plasmon. We show that in the case of $1 \mathrm{D}$ periodic substrate the contribution of higher harmonics to electromagnetic fields vanishes in the long-wavelength limit. A specific feature of the Kronig-Penney model for plasmonic crystal is independence of the resonant frequency of surface plasmon of dielectric properties of the substrate. This is due to zero filling fraction of the dielectric component.

\section{ACKNOWLEDGMENT}

This work was supported by the U.S. Department of Energy under Grant No. DE-FG02-06ER46312.
${ }^{1}$ E. N. Economou, Phys. Rev. 182, 539 (1969).

${ }^{2}$ D. Sarid, Phys. Rev. Lett. 47, 1927 (1981).

${ }^{3}$ H. Raether, Surface Plasmons (Springer-Verlag, Berlin, 1988); V. M. Agranovich, Surface Polaritons, edited by D. L. Mills, (North-Holland, Amsterdam, 1982); G. J. Kovacs, Thin Solid Films 60, 33 (1979); E. Kretschmann, Opt. Commun. 10, 353 (1974); Electromagnetic Surface Modes, edited by A. D. Boardman, (Wiley, New York, 1982); R. H. Ritchie, Phys. Rev. 106, 874 (1957); H. Raether, in Physics of Thin Films, edited by G. Haas, M. H. Francombe, and R. W. Hoffmann, (Academic, New York, 1977), Vol. 9, p. 145.

${ }^{4}$ W. L. Barnes, J. Opt. A, Pure Appl. Opt. 8, S87 (2006).

${ }^{5}$ P. Berini, Phys. Rev. B 61, 10484 (2000); 63, 125417 (2001); R. Buckley and P. Berini, Opt. Express 15, 12174 (2007).

${ }^{6}$ J. Q. Lu and A. A. Maradudin, Phys. Rev. B 42, 11159 (1990); I. V. Novikov and A. A. Maradudin, ibid. 66, 035403 (2002).

${ }^{7}$ S. I. Bozhevolnyi, V. S. Volkov, E. Devaux, and T. W. Ebbesen, Phys. Rev. Lett. 95, 046802 (2005); S. I. Bozhevolnyi, V. S.
Volkov, E. Devaux, J. Y. Laluet, and T. W. Ebbesen, Nature (London) 440, 508 (2006); T. W. Ebbesen, C. Genet, and S. Bozhevolnyi, Phys. Today 61, 44 (2008).

${ }^{8}$ A. Neogi, Ch. W. Lee, H. O. Everitt, T. Kuroda, A. Tackeuchi, and E. Yablonovitch, Phys. Rev. B 66, 153305 (2002); K. Okamoto, I. Niki, A. Shvartser, Y. Narukawa, T. Mukai, and A. Scherer, Nature Mater. 3, 601 (2004).

${ }^{9}$ T. Taubner, D. Korobkin, Y. Urzhumov, G. Shvets, and R. Hillenbrand, Science 313, 1595 (2006).

${ }^{10}$ M. Liu, M. Pelton, and P. Guyot-Sionnest, Phys. Rev. B 79, 035418 (2009).

${ }^{11}$ R. Li, C. Cheng, Fang-Fang Ren, J. Chen, Ya-Xian Fan, J. Ding, and H. T. Wang, Appl. Phys. Lett. 92, 141115 (2008).

${ }^{12}$ V. N. Konopsky and E. V. Alieva, Phys. Rev. Lett. 97, 253904 (2006).

${ }^{13}$ A. A. Krokhin, A. Neogi, and D. McNeil, Phys. Rev. B 75, 235420 (2007).

${ }^{14}$ J. Jacob, A. Babu, G. Mathew, and V. Mathew, Superlattices 
Microstruct. 44, 282 (2008).

${ }^{15}$ P. Halevi, A. A. Krokhin, and J. Arriaga, Phys. Rev. Lett. 82, 719 (1999).

${ }^{16}$ A. V. Zayats, I. I. Smolyaninov, and A. A. Maradudin, Phys. Rep. 408, 131 (2005).

${ }^{17}$ J. A. Dionne, L. A. Sweatlock, H. A. Atwater, and A. Polman, Phys. Rev. B 73, 035407 (2006).

${ }^{18}$ F. Pincemin, A. A. Maradudin, A. D. Boardman, and J.-J. Greffet, Phys. Rev. B 50, 15261 (1994).

${ }^{19}$ J. A. Dionne, E. Verhagen, A. Polman, and H. A. Atwater, Opt. Express 16, 19001 (2008).

${ }^{20}$ M. M. Doria, F. Parage, and O. Buisson, Europhys. Lett. 35, 445
(1996).

${ }^{21}$ F. G. Elmzughi, J. Phys.: Condens. Matter 7, 7023 (1995).

${ }^{22}$ G. P. Ortiz, B. E. Martínez-Zérega, B. S. Mendoza, and W. L. Mochán, Phys. Rev. B 79, 245132 (2009); V. Cerdán-Ramírez, B. Zenteno-Mateo, M. P. Sampedro, M. A. Palomino-Ovando, B. Flores-Desirena, and F. Pérez-Rodríguez, J. Appl. Phys. 106, 103520 (2009).

${ }^{23}$ I. I. Smolyaninov, Appl. Phys. A: Mater. Sci. Process. 87, 227 (2007).

${ }^{24}$ J. Elser and V. A. Podolskiy, Phys. Rev. Lett. 100, 066402 (2008). 